Proc. of 12th International Workshop on Positron and Positronium Chemistry, August 28-September 1, 2017, Lublin, Poland

\title{
Gamma Induced Positron Annihilation: History, Current, and Future Developments
}

\begin{abstract}
F. SELIM ${ }^{a, b}$
${ }^{a}$ Department of Physics and Astronomy, Bowling Green State University, Bowling Green, Ohio 43403, USA

${ }^{b}$ Center of Photochemical Sciences, Bowling Green State University, Bowling Green, Ohio 43403, USA

Positron annihilation spectroscopy is often performed using radioactive sources for bulk measurements or positron beams for depth resolved measurements. Both have many advantages and great capabilities for a variety of applications. In the recent history, we have shown that positron annihilation spectroscopy can be carried out directly using high energy photons without the need for positron source or positron beam. This approach brings unique capabilities for some specific applications and promotes the use of positron annihilation spectroscopy in new areas of materials science and probably in industrial applications. Some of the important applications include developing new nondestructive highly penetrating sensitive probe for structural and engineering materials. It can also greatly advance positron applications in bulk semiconductors, electronic and photonic materials as well as in polymers, ceramics, and liquids. The recently developed $\gamma$-induced positron spectroscopy in HZDR in Dresden provides an example of an excellent facility for many of these applications. When incorporated with pulsed accelerators, $\gamma$-induced positron annihilation spectroscopy may trigger novel studies of transient states in matter and explore several solid-state processes that take place on short time scale. In this article I will review the history and development of the technique and its incorporation in a wide range of accelerators including table top electron accelerators, pulsed electron accelerators, and Van de Graaff accelerators. Then I will introduce a design for a new $\gamma$-induced positron annihilation spectroscopy facility based on using small nuclear research reactors or neutron generators. The paper presents all the possible approaches for $\gamma$-induced positron annihilation spectroscopy and discusses its potential and limitations to guide the efforts in further development of the technique and illustrate the unique aspects that the technique can bring to positron science and applications.
\end{abstract}

DOI: $10.12693 /$ APhysPolA.132.1450

PACS/topics: gamma induced positron spectroscopy, positron transient measurements, thermal neutron capture, proton capture, electron accelerator, Van de Graaff accelerator

\section{Introduction}

Positron annihilation spectroscopy (PAS) has been established as an effective tool for material characterization and defect studies [1-3]. During the last 5 decades, positron annihilation lifetime spectroscopy (PALS), the Doppler broadening of positron annihilation spectroscopy (DBPAS), and angular correlation of annihilation radiation (ACAR) techniques have been developed and significantly contributed to a broad range of science in metals, semiconductors, and polymers. Bulk measurements have been always carried out using radioactive source, mostly Na-22 and positron beams have been developed worldwide to provide a powerful tool for depth resolved measurements [4]. Recently we have shown the possibility of performing both PALS and DBPAS using high energy gamma rays produced from various types of accelerators [5-7]. This approach offers specific advantages and initiates new applications for PAS. It is not a replacement for bulk positron measurements using radioactive source or positron beam applications, however it promotes new positron applications, solves some of its technical problems and provides a tool for positron transient measurements as well as it may facilitate the application of PAS in industry.

The principle of $\gamma$-induced PAS is based on using high energy gamma rays to generate positrons directly inside the sample by pair production and performing PALS, DBPAS, or ACAR measurements; it eliminates the need for positron source or positron beam. The energy of $\gamma$ - rays should be above $1.02 \mathrm{MeV}$ for the pair creation to take place, which dramatically increases with increase of $\gamma$-ray energy. However, the $\gamma$-energy should be maintained below 10-15 MeV, the neutron emission threshold in most materials to avoid inducing radioactivity and atom recoil in the sample following neutron emission in $(\gamma, \mathrm{n})$ reaction. The positron production rate and the $511 \mathrm{keV}$ detection efficiency strongly depend on the atomic number of the samples as the pair production cross-section is proportional to $Z^{5}$, where $Z$ is the sample atomic number [8]. Beam hardening and collimation of $\gamma$-rays before bombarding the sample are crucial to reduce background and enable $\gamma$-spectroscopy. $\gamma$-ray penetrate deeply in materials providing highly penetrating probe. The technique is limited to bulk measurements, but it brings unique capabilities and extends PAS to new applications such as high sensitivity defect spectroscopy in thick materials and study of solvated electrons and positron annihilation in solutions. It provides a perfect method for lifetime measurements in bulk materials free of source and other unwanted contributions and thereby advancing the capability of positron in identifying more defect species in materials. These new capabilities with several other advantages will be discussed in this article in detail.

$\gamma$-rays can be generated from various sources. In case of electron accelerators, a tungsten converter can be used to generate high energy bremsstrahlung radiation. However, bremsstrahlung radiation has high level of useless 
low energy photons $<1.02 \mathrm{MeV}$ which do not contribute to positron production; they must be suppressed by beam hardening through a low atomic number material such as aluminum target. At least two stages collimation is necessary in case of using bremsstrahlung radiation from accelerators to reduce background at the sample station. Nuclear reactions represent another source for $\gamma$-rays; they provide nearly mono-energetic prompt $\gamma$-rays and significantly reduce background radiation in $\gamma$-induced PAS. DBPAS and ACAR measurements can be performed using any high intense $\gamma$-rays while PALS require short pulsed accelerators or coincident $\gamma$-rays commonly originated in nuclear reactions. Number of works $[5,6,9]$ have confirmed the feasibility of DBPAS and PALS using $\gamma$-ray, however $\gamma$-induced ACAR measurements have yet to be performed.

In this study, all the possible gamma-induced positron annihilation spectroscopy approaches will be presented and the advantages and limitations of the technique will be discussed. The paper addresses potential new science that may emerge with this technique. It discusses dynamic measurements of electron positron annihilation through synchronizing pulsed bremsstrahlung radiation with laser pulses and illustrates the technique capabilities in studying excited and transient states in matter. The paper also presents a new approach for PAS based on using nuclear reactors or neutron generators to generate $\gamma$-rays through thermal neutron capture. This approach would overcome the problems of high background radiation and low count rate associated with the use of accelerators as thermal neutron capture reactions provides prompt monoenergetic $\gamma$-rays and may substantially reduce background. Small nuclear research reactors have limited utility in recent decades; the technique presented here would create new capabilities and rejuvenate research activities at these small reactors.

\section{2. $\gamma$-induced PAS techniques}

\subsection{DBPAS using small electron accelerators}

Early experiments [5, 10, 11] illustrated the possibility of performing DBPAS using $6 \mathrm{MeV}$ electrons from $200 \mathrm{HZ}$ small linear electron accelerator (Linac). A schematic diagram of the setup is shown in Fig. 1 and an example of the measured spectrum is given in Fig. 2. From the analysis of the $511 \mathrm{keV}$ annihilation peak, the defect parameters $S$ and $W$ can be evaluated [2]. The technique was applied for stress analysis in thick steel samples (Fig. 3a), however the low repetition rate of the Linac used in this experiment led to high statistical errors in $S$-parameter as shown in Fig. 3b, which displays the $S$-parameter as a function of strain for the thick steel samples (shown in Fig. 3a). Despite the high statistical errors, the measurements were successful to illustrate the capability of $\gamma$ induced PAS in measuring stress at high depths in thick materials. By comparing the $S$-parameter-strain curve with the stress-strain engineering curve in Fig. 3b, it can be seen that the defect parameter $S$ exhibits similar behavior to the stress-strain engineering curve of materials.
Several experiments [12-14] have been performed to measure the induced stress due to tensile, fatigue, cold work, and bending tests using this method. These measurements illustrate that $\gamma$-induced PAS can be developed to an effective tool for inspection of structural and engineering materials. Further development of the technique requires the use of high repetition rate accelerators or continuous-wave (CW) electron beams, which would increase the count rate million times and significantly improve the statistics.

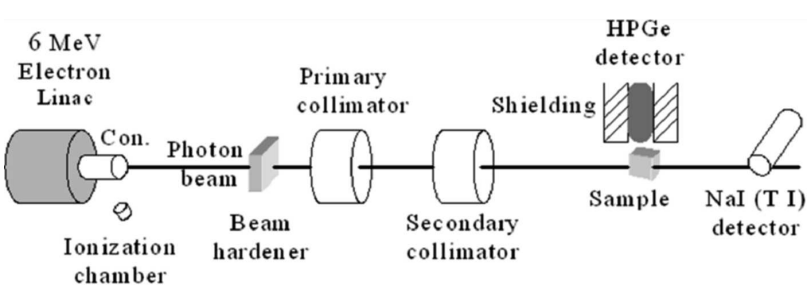

Fig. 1. A schematic diagram of $\gamma$-ray induced PAS by means of small pulsed electron accelerator.

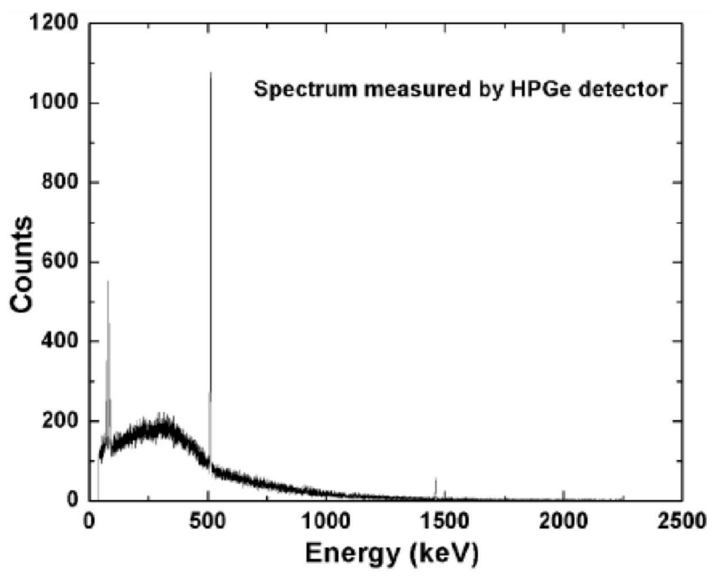

Fig. 2. Doppler broadening spectrum of annihilation radiation emitted from a lead target in $\gamma$-induced PAS. The first peak in the spectrum is due to $K_{\alpha} \mathrm{X}$-ray lines from the lead shielding around the detector.

\subsection{Proton capture induced PAS}

Another highly effective method to carry out both $\gamma$ induced PALS and $\gamma$-induced DBPAS is based on using proton beams from Van de Graaff accelerator to obtain monoenergetic $\gamma$-rays from proton capture in light elements. This concept has been tested using ${ }^{27} \mathrm{Al}(\mathrm{p}, \gamma){ }^{28} \mathrm{Si}$ reaction, which results in 2 coincident $\gamma^{\prime}$ s of $2.8 \mathrm{MeV}$ and $1.78 \mathrm{MeV}$ providing a convenient route for positron lifetime measurements. The reaction has a strong resonance at $992 \mathrm{keV}$ proton energy [15], which leads to high efficiency $\gamma$-production at $1 \mathrm{MeV}$ proton energy. First experiment [6] was illustrated by focusing a $1.5 \mathrm{MeV}$ proton beam of $0.2 \mu \mathrm{A}$ from a Van de Graaff accelerator onto a thin $\mathrm{Al}$ window mounted at the end of the proton 


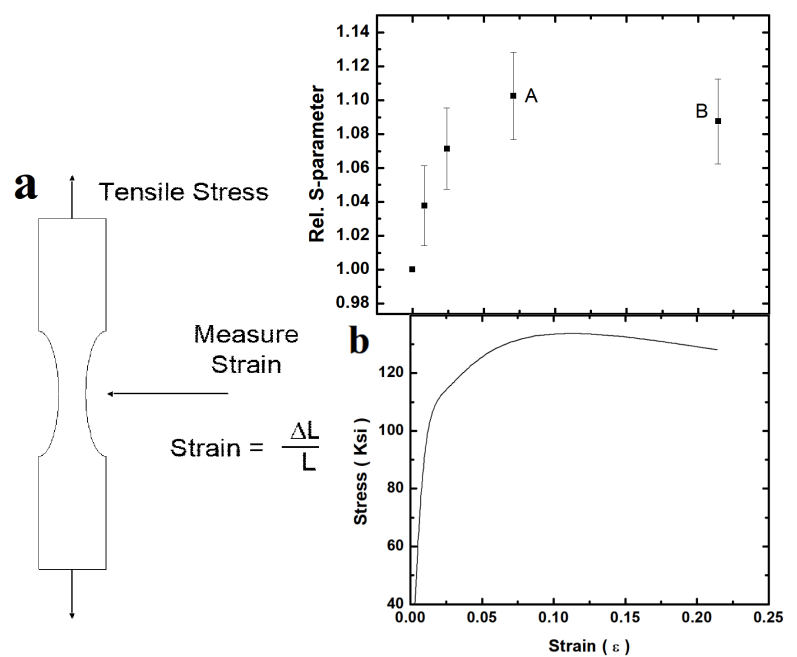

Fig. 3. Thick steel specimen used for stress strain measurements. The arrows show the direction of applied stress and the area where the strain was measured. (b) Comparison between the $S$ parameter-strain curve and the stress-strain curve for the specimen shown in Fig. 3a.

beam line. ${ }^{27} \mathrm{Al}(\mathrm{p}, \gamma){ }^{28} \mathrm{Si}$ reaction populates high energy excited states in Si that decay to the first excited state emitting prompt $\gamma$-rays of $2.8 \mathrm{MeV}$ or $3.2 \mathrm{MeV}$. Then a decay to the ground state takes place within 475 fs emitting a $1.78 \mathrm{MeV} \gamma$-photon (Fig. 4). Figure 5 shows an illustration for PALS concept using this reaction, where the $1.78 \mathrm{MeV}$ photon is used to start the clock giving an indication for positron birth and the $2.8 \mathrm{MeV}$ photon is sent to the sample generating a positron inside it. The stop signal is obtained by recording one of the annihilation pairs. PALS measurements using this setup were carried out in annealed $\mathrm{Pb}, \mathrm{Al}$ and $\mathrm{Cu}$ targets using proton capture in $\mathrm{Al}$ window and reveal single positron decay curve with no source contribution or background [6]. In standard bulk PALS, about $10 \%-15 \%$ of positrons annihilate in the source, cladding materials and the interface between the sample surface and source foil. This distorts the positron lifetime spectra, complicates the data analysis, and limits the number of defect species that can be identified in the sample. $\gamma$-induced PALS by proton captures eliminates all these problems and provides clean positron lifetime spectrum free of source contributions, which greatly facilitates spectrum fitting and extracting lifetime components and allows one to identify more defect species in the sample. Recently, DBPAS has been successfully performed by Pujari et al. [16] to study defects in large samples of metals and polymers using the proton capture reaction ${ }^{19} \mathrm{~F}\left({ }^{1} \mathrm{H}, \gamma\right){ }^{16} \mathrm{O}$ in fluorine.

\section{3. $\gamma$-induced PAS using pulsed superconducting electron accelerators}

The use of superconducting electron accelerators that generates intense pulsed bremsstrahlung radiation solves

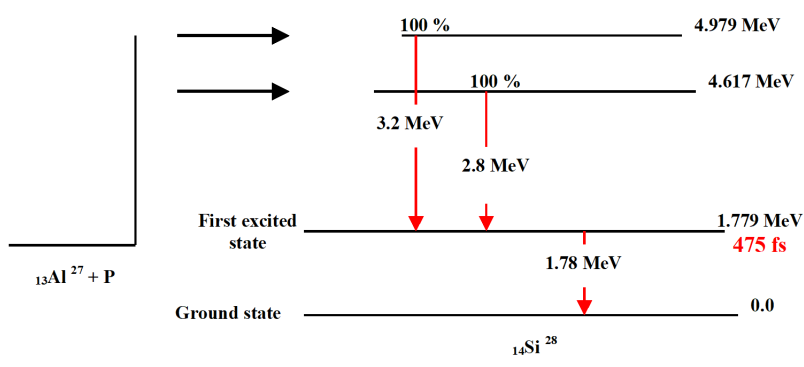

Fig. 4. A schematic diagram for the proton capture process in Al-27 and the decay of Si-28 illustrating the production of coincident $\gamma$-photons.

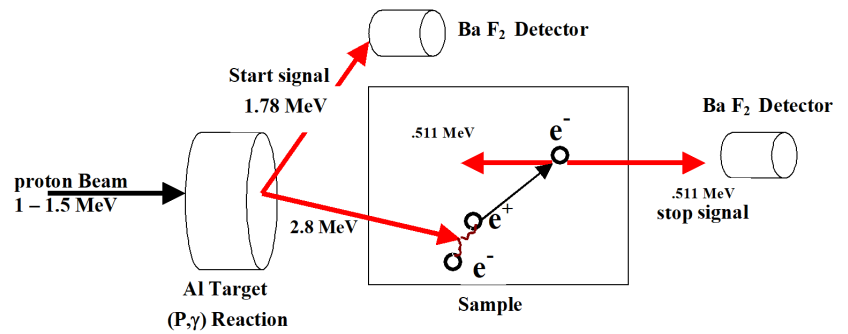

Fig. 5. An illustration of the concept of positron lifetime spectroscopy by proton capture in Al-target.

the problem of low counting rate and high statistical errors associated with small electron accelerators. This has been achieved by developing the unique facility "Gamma Induced Positron Spectroscopy (GIPS)" at HelmholtzZentrum Dresden-Rossendorf (HZDR) in Dresden, Germany $[9,17]$. The HZDR superconducting accelerator runs at $26 \mathrm{MHz}$ providing a great platform to perform positron lifetime measurements. Since its development, GIPS has been used to carry out positron measurements in a wide range of systems $[18,19]$ bringing new capabilities such as performing positron measurements in solutions, chemicals, and radioactive samples and enhancing defect analysis in semiconductors and oxides by eliminating source contribution and background from lifetime spectra [19].

\section{4. $\gamma$-induced PAS using ultrashort laser-Compton scattered gamma ray pulses}

Ultrashort high energy $\gamma$-ray pulses generated by laser Compton scattering with $90^{\circ}$ collisions between an electron beam and a femtosecond laser at the UVSOR-II electron storage ring in Tsukuba, Japan has been recently used for PALS measurements in thick materials [20]. The developed spectrometer achieved remarkable time resolution of $88 \mathrm{ps}$ in thick lead target. More details about the measurements can be found in Ref. [20].

\section{5. $(n, \gamma)$ reaction induced $P A S$}

Intense electron beams from large accelerators generate tremendous intense bremsstrahlung radiation, which 
dramatically increases count rates and enables the practical use of the $\gamma$-induced PAS. However, bremsstrahlung radiation also carry immense contributions from low energy photons which is not useful for pair production but creates high background radiation hindering the detector performance and limits our capability in improving the count rate and reducing measuring time. The use of monoenergetic gamma rays in the range of 8 to $10 \mathrm{MeV}$ instead of bremsstrahlung radiation to generate positrons inside the sample by pair production would greatly reduce the background and significantly advance the technique and its applications.

We have recently designed $\gamma$-induced PAS facility based on the use of thermal neutron beam, where highenergy gamma rays in the range of several $\mathrm{MeV}$ are generated by thermal-neutron capture in $\mathrm{Fe}, \mathrm{Ni}$, or Gd. Capture of thermal neutrons in any of these targets leads to emission of prompt $\gamma$-rays through $(\mathrm{n}, \gamma)$ reactions and the target thereby radiates prompt $\gamma$-rays in all directions. Gd can be a very effective $\gamma$-radiator as Gd-157 has the highest thermal neutron capture cross-section among all stable nuclei [21]. Gd-155 has also high cross-section; the two isotopes generate $\gamma$-ray around $8 \mathrm{MeV}$ through the two reactions: ${ }^{155} G d+n \rightarrow{ }^{156} G d+\gamma(8.5 \mathrm{MeV})$ and ${ }^{157} \mathrm{Gd}+\mathrm{n} \rightarrow{ }^{158} \mathrm{Gd}+\gamma(7.9 \mathrm{MeV})$. The abundance of the two isotopes is about $40 \%$. Fe may be also a good radiator as thermal neutron capture in Fe-56, the most abundant iron isotope, leads to the emission of several prompt lines with the highest yield at $7.6 \mathrm{MeV}$. Highenergy gamma rays enhance positron production since the pair-production cross-section increases exponentially with energy above the threshold energy of $1.022 \mathrm{MeV}$. Both $\gamma$-induced Doppler broadening and lifetime spectrometers can be developed using this approach as the coincident prompt $\gamma$ 's emitted from $(\gamma, \mathrm{n})$ reactions provides an easy platform for PALS measurements. The spectrometer has been designed to be attached to the thermal neutron port of the $1 \mathrm{MW}$ research reactor at Washington State University in USA. Figure 6 displays the measured thermal neutron flux after filtration at the thermal neutron port of the reactor illustrating the possibility of obtaining high thermal neutron flux from a small nuclear reactor.

Figure 7 shows the overall design of the photon beam line and spectrometer, including four gamma-ray detectors, two having $\mathrm{BaF}_{2}$ scintillators and two of high-purity Ge (HPGe), to be used, respectively, for the positron lifetime and the Doppler broadening measurements. A boron block is mounted just in front of the radiator to absorb any remaining neutrons in order to prevent them from reaching the sample chamber or detectors. A large lead shield is constructed around the radiator to reduce emission of $\gamma$-rays in the surroundings. The $\gamma$-ray beam is transmitted to the sample chamber after passing through a beam hardener made of aluminum to absorb low-energy photons and two lead collimators to limit the diameter of the photon beam at the target in order to reduce background radiation around the detectors. Gamma rays

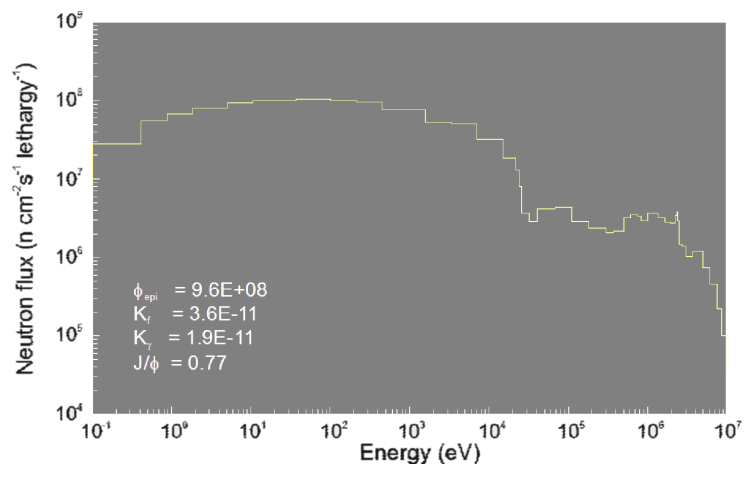

Fig. 6. Distribution of thermal neutron flux after removing high energy neutrons at the external port of the WSU nuclear reactor. High thermal neutron flux is essential for efficient generation of prompt $\gamma$-rays from nuclear reactions.

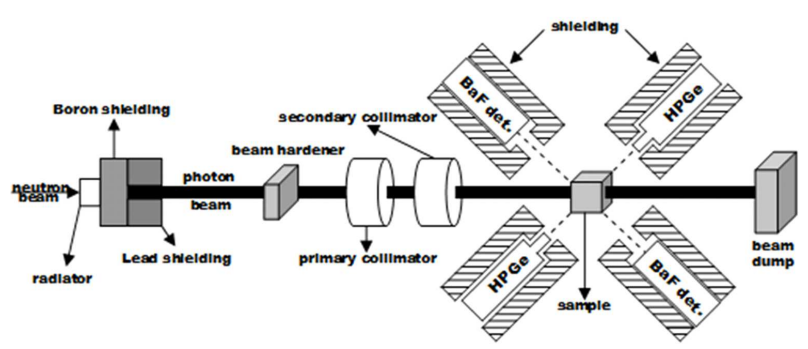

Fig. 7. Design of the $\gamma$-induced PAS facility built on thermal neutron capture and $\gamma-\mathrm{n}$ reaction.

transmitted through the sample are stopped away from the detectors to reduce contributions from scattered photons or unwanted $511 \mathrm{keV}$ radiation in the detectors and a $\mathrm{NaI}$ detector is used to monitor the incident photon beam intensity. The photon beam intensity and subsequent positron production rate and detection efficiency can be adjusted by controlling the mass of the radiator.

A great alternative for this nuclear reactor based $\gamma$ induced PAS may be available fairly soon as the recent development in neutron generators, deuteriumdeuterium (D-D) and deuterium-tritium ( $\mathrm{D}-\mathrm{T})$ generators provides a practical source for generating neutrons anywhere. These recently developed generators currently generate up to $10^{13}$ thermal neutrons flux, the typical neutron beam intensity in most research reactors. The use of neutron generators may facilitate the incorporation of $\gamma$-induced PAS spectrometer in any facility and promote the use of positron annihilation in industry.

\section{Advantages and limitations of $\gamma$-induced PAS}

$\gamma$-induced PAS combines the high sensitivity of positrons to open volume defects with the high penetration of $\gamma$-rays providing highly penetrating sensitive nondestructive probe for defect and stress measurements in thick materials. Such technique is highly desirable for testing engineering and structural materials. In fact, 
most of the current nondestructive techniques are limited by either small penetration depth or low sensitivity $[22,23]$. Furthermore, the use of small accelerators or neutron generators eliminates the hassle and the safety issues associated with the use of radioactive sources, which is one of the reasons that hinders positron applications in industry. With respect to PALS studies of defects, $\gamma$-ray induced PAS in bulk materials eliminates the unwanted contributions in positron lifetime spectra due to positron annihilation in the source material, cladding materials and interfaces. These contributions account for about 10 to $15 \%$ in the spectra and often complicate data analysis and restrict the number of defect types that can be extracted from PALS measurements [24]. The exact source contribution in the spectra differs from run to run based on the geometry, sample nature and surface, etc. and it is sometimes hard to estimate its exact contribution in lifetime spectra raising concern about the validity of the extracted lifetime components. By eliminating these contributions from lifetime measurements, $\gamma$-induced PAS provides clean lifetime spectra and allow the identification of more defect types in the sample, which will greatly advance positron spectroscopy of defects in semiconductors and oxides. Our recent measurements [19] of lifetime spectra in small $\mathrm{ZnO}$ crystals of few mm thickness in GIPS facility proved that the technique is effective in studying defects in semiconductors even in small thin samples.

The technique facilitates PAS measurements in many avenues where source based PAS is complicated or even not possible. For example, study of solvated electrons and positron annihilation in chemicals and liquids is a challenge because $\mathrm{Na}-22$ source often dissolves in liquids leading to safety concerns and making PAS studies of chemicals unattainable. PAS at high temperature is currently not possible because of the evaporation of Na-22, which hampers our capability in measuring electron states and excited states at high temperatures or dynamic states of defects. On the contrary, $\gamma$-induced PAS would grant a simple way for such studies in liquids and bulk materials. Another example which could significantly benefit from $\gamma$-induced PAS is radiation damage and defect measurements in radioactive materials. The emitted $\gamma$-ray from radiative samples interfere with Na-22 emission distorting lifetime spectra; thus a complicated experimental setup of 3 detectors and sophisticated data analysis are imperative for this kind of investigation. Study of defects in radioactive materials is of great importance in nuclear materials and fission and fusion technologies and $\gamma$-induced PAS represents a unique tool for these studies. Lastly PAS measurements during excitation and transient states can be done effectively using $\gamma$-ray from pulsed electron accelerators as demonstrated below. $\gamma$-induced PAS is limited to the investigation of bulk materials and it cannot be developed to a local probe or depth resolved technique. This inhibits its applications in thin films investigation and depth dependent defect studies.

\section{Measurements of dynamic states of positron annihilation}

$\gamma$-induced PAS offers a convenient way to carry out dynamic states of electron-positron annihilation measurements by synchronizing bremsstrahlung pulses from pulsed electron accelerators with short pulsed laser irradiation. Figure 8 illustrates a schematic diagram for

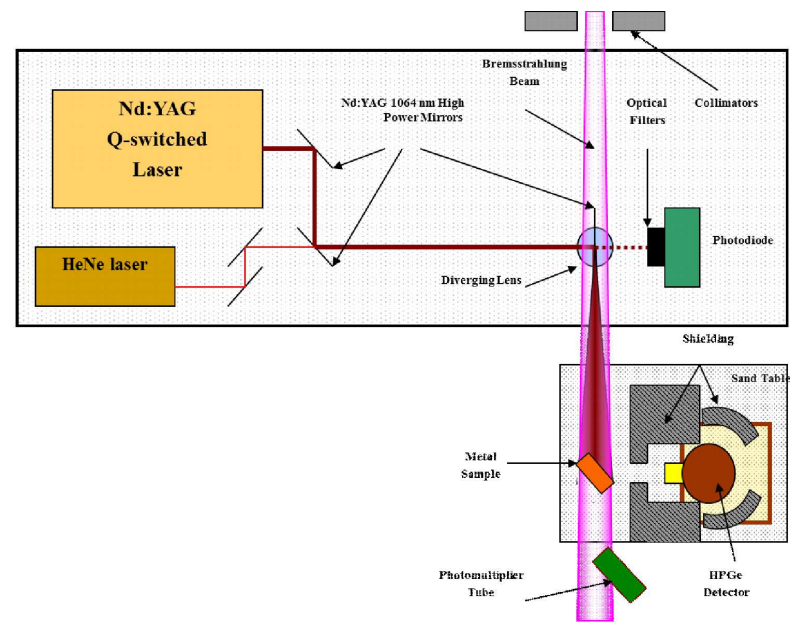

Fig. 8. Dynamic PAS measurements setup. The figure shows the synchronization between laser pulses from high power Nd-YAG laser system and bremsstrahlung pulses from $20 \mathrm{MeV}$ pulsed electron accelerator.

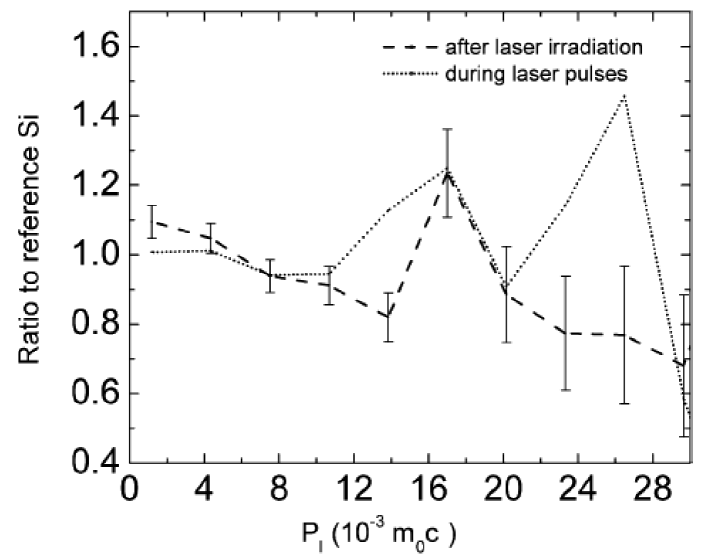

Fig. 9. Doppler broadening measurements of the longitudinal electron momentum distributions in single crystal silicon during laser pulses and after laser irradiation.

$\gamma$-induced PAS synchronized with short laser pulses for transient studies. The experiment was carried out on a $20 \mathrm{MeV}$ pulsed electron accelerator at the Idaho Accelerator Center in USA [7]. The pulsed electron beam was converted through a tungsten converter to bremsstrahlung pulses and synchronized with laser pulses from a high power Nd:YAG laser source. To achieve synchronization, the laser source was triggered by a signal from the circuit that drives the electron gun of the accelerator. The 
pulse temporal width is 8 ns for laser pulses and can be controlled between 7 and 40 ns for bremsstrahlung photon pulses. A photodiode was used to measure the power of the laser pulses. It also detects each laser pulse and sends a signal to a constant fraction discriminator (CFD), while a $\mathrm{BaF}_{2}$ detector was used to detect the bremsstrahlung pulse and send a signal to another CFD. The first CFD provides a start signal to a time-amplitude converter (TAC) and the second CFD provides a stop signal and the timing spectrum generated from the TAC was used to monitor and adjust the synchronization between the laser pulses and bremsstrahlung pulses. The setup was used to study electron states in silicon during laser excitation by ultraviolet, visible and infrared waves [7]. The original laser wavelength was $1064 \mathrm{~nm}$ and both $532 \mathrm{~nm}$ and $266 \mathrm{~nm}$ pulses were obtained by second and third harmonic generation. Figure 9 presents the ratio curve of the longitudinal electron momentum distribution in Si during and between $1064 \mathrm{~nm}$ laser pulses. The ration curve was obtained with respect to reference Si. This experiment illustrated a convenient way for dynamic measurements of positron annihilation.

\section{Summary}

The paper presents a comprehensive review on $\gamma$ induced PAS and all the various approaches in generating intense high energy $\gamma$-rays for positron production directly inside the target. These approaches include nuclear reactions such as proton capture and neutron capture, high energy electron beams from accelerators and the Compton scattering of electron beam by laser. The paper also illustrates the advantages and limitations of the technique and its potential in boosting new applications and facilitating dynamic measurements of positron annihilation in matter under excitation and extreme conditions. A new technique PAS based on employing nuclear reactors or neutron generators has been presented to generate cleaner high energy photon beams which will substantially reduce background and improve detection efficiency. This approach can take advantages of the underused nuclear reactors and connect nuclear science with material science while boosting new research tools for a wide range of materials.

\section{Acknowledgments}

The author would like to thank Don Wall and MichałSilarski for their valuable inputs.

\section{References}

[1] S. Kahana, Phys. Rev. 117, 123 (1960).

[2] R. Krause-Rehberg, H.S. Leipner, Positron Annihilation in Semiconductors: Defect Studies, Springer Science, 1999, p. 127.

[3] Y.C. Jean, Mater. Sci. Forum 175-178, 59 (1995).
[4] P.J. Schultz, K.G. Lynn, Rev. Mod. Phys. 60, 701 (1988).

[5] F. Selim, D. Wells, J. Harmon, J. Williams, J. Appl. Phys. 97, 113539 (2005).

[6] F. Selim, D. Wells, J. Harmon, Rev. Sci. Instrum. 76, 033905 (2005).

[7] F. Selim, Phys. Lett. A 344, 291 (2005).

[8] C. Davisson, in: Alpha-, Beta- and Gamma-Ray Spectroscopy, 1965, p. 37.

[9] M. Butterling, W. Anwand, T.E. Cowan, A. Hartmann, M. Jungmann, R. Krause-Rehberg, A. Krille, A. Wagner, Nucl. Instrum. Methods Phys. Res. B 269, 2623 (2011).

[10] F. Selim, D. Wells, J. Harmon, W. Scates, J. Kwofie, R. Spaulding, S. Duttagupta, J. Jones, T. White, T. Roney, Nucl. Instrum. Methods Phys. Res. B 192, 197 (2002).

[11] F. Selim, D. Wells, F. Harmon, J. Kwofie, G. Lancaster, J. Jones, in: AIP Conf. Proc., AIP, 2003, p. 499.

[12] F. Selim, D. Wells, J. Harmon, J. Williams, J. Appl. Phys. 97, 113540 (2005).

[13] F. Selim, D. Wells, J. Harmon, J. Kwofie, A. Roy, T. White, T. Roney, Adv. X-ray Anal. 46, 106 (2002).

[14] F. Selim, D. Wells, J. Harmon, J. Kwofie, G. Erikson, T. Roney, Radiat. Phys. Chem. 68, 427 (2003).

[15] R. Bondelid, C. Kennedy, Phys. Rev. 115, 1601 (1959).

[16] P. Pujari, K. Sudarshan, R. Tripathi, D. Dutta, P. Maheshwari, S. Sharma, D. Srivastava, R. KrauseRehberg, M. Butterling, W. Anwand, Nucl. Instrum. Methods Phys. Res. B 270, 128 (2012).

[17] M. Butterling, W. Anwand, G. Brauer, T.E. Cowan, A. Hartmann, M. Jungmann, K. Kosev, R. KrauseRehberg, A. Krille, R. Schwengner, Physica Status Solidi A 207, 334 (2010).

[18] S.M. Beck, M. Butterling, W. Anwand, A. Beck, A. Wagner, G. Brauer, A. Ocherashvili, I. Israelashvili, O. Hen, in: J. Phys. Conf. Series 443, 012076 (2013).

[19] J. Ji, A. Colosimo, W. Anwand, L.A. Boatner, A. Wagner, P. Stepanov, T. Trinh, M. Liedke, R. Krause-Rehberg, T. Cowan, Sci. Rep. 6, 31238 (2016).

[20] Y. Taira, H. Toyokawa, R. Kuroda, N. Yamamoto, M. Adachi, S. Tanaka, M. Katoh, Rev. Sci. Instrum. 84, 053305 (2013).

[21] G. Leinweber, D.P. Barry, M. Trbovich, J. Burke, N. Drindak, H. Knox, R. Ballad, R. Block, Y. Danon, L. Severnyak, Nucl. Sci. Eng. 154, 261 (2006).

[22] G. Dobmann, N. Meyendorf, E. Schneider, Nucl. Eng. Des. 171, 95 (1997).

[23] I.C. Noyan, J.B. Cohen, Residual Stress: Measurement by Diffraction and Interpretation, Springer, 2013.

[24] K. Saarinen, P. Hautojärvi, C. Corbel, Semicond. Semimet. 51, 209 (1998). 